\title{
Optical reproduction of sounds from old phonographic wax cylinders
}

Takashi Nakamura, T. Ushizaka, Jun Uozumi, Toshimitsu Asakura

Takashi Nakamura, T. Ushizaka, Jun Uozumi, Toshimitsu Asakura, "Optical reproduction of sounds from old phonographic wax cylinders," Proc. SPIE 3190, Fifth International Topical Meeting on Education and Training in Optics, (8 December 1997); doi: 10.1117/12.294398

Event: Fifth International Topical Meeting on Education and Training in Optics, 1997, Delft, Netherlands 


\title{
Optical reproduction of sounds from old phonographic wax cylinders
}

\author{
T. Nakamura \\ Kushiro National College of Technology \\ Kushiro, Hokkaido, 084 Japan \\ T. Ushizaka and J. Uozumi \\ Research Institute for Electronic Science, Hokkaido University \\ Sapporo, Hokkaido, 060 Japan \\ T. Asakura \\ Faculty of Engineering, Hokkai-Gakuen University \\ Sapporo, Hokkaido, 064 Japan
}

\begin{abstract}
It is shown that reproduction of sounds from old wax cylinders using various optical methods gives quite excellent materials in optics education. The laser-beam reflection method is explained by the geometrical optics, and is further discussed on the basis of the diffraction theory. Various techniques for improving the quality of reproduced sounds are introduced. Fiber optics is used for reproduction of sounds in a low pressure stylus contacting method. Applications of the laser-beam reflection method to other analogue recordings are introduced.
\end{abstract}

\section{INTRODUCTION}

Nowadays, the optical technology plays a major role in the field of recording media such as the compact disk, magneto-optical disk, and digital versatile disk. It seems that compact disks have replaced analogue disk records almost completely. It is also recognized, however, that some people, especially in young generations, show strong interest in sounds from traditional analogue records. This is no doubt due to a rebound from highly sophisticated digital technology to more simple and classic methods. People always express interest in the origin of a technology even in the high-tech age.

Therefore, it would stimulate curiosity of students to relate a modern technology with its classic version. A highly attractive material from this point of view is the application of laser technology to reproduction of sounds from old phonographic wax cylinders. This material would afford the understanding of both the classic recording method and the basic principle for handling a laser beam to detect sound signals from recording materials.

The phonographic wax cylinder invented by Thomas Edison in 1885 was used for recording and reproducing sounds until about 1930 . During these years, numerous recordings were made for various purposes, for example, in daily life scenes, in amusements of early jukeboxes, and in artistic recordings of famous musicians. Some of those cylinders have remained until now and become an important cultural inheritance.

Although the reproduction of sounds from such old wax cylinders is readily realized by using the Edison-type phonograph, this method usually causes serious damages on sound grooves of wax cylinders because of the heavy stylus pressure. The laser-beam reflection method ${ }^{1}$ was first developed at Hokkaido University for the purpose of non-destructive reproduction of sounds from Piłsudski's wax 
cylinders discovered at Mickiewicz University in Poland. This method was successfully used for reproducing sounds from wax cylinders and the talks and songs of Ainu people in Sakhalin and Hokkaido were clearly reproduced. This work has made a great contribution to both philology and folklore of the Ainu people.

While this development was completed successfully, this work has been continued to improve the quality of reproduced sounds from wax cylinders. Through the improvement of the laser-beam reflection method, it is found that this material provides various educational stages for beginners in optics. In some cases, the optical configuration employed is so simple that it is readily set up by beginners in optics, and reproduced sounds attract much interest of them.

There are mainly two types of improvements. One is to proceed the non-contacting technique of the laser-beam reflection method. The other is to develop a method of a contacting technique with acceptable little damages.

In this paper, principles of the laser-beam reflection method are first described in detail for the basis of the reproduction of sounds from old wax cylinders. Problems and shortcomings of the method and their solutions are explained in relation to the viewpoint of optics education. Furthermore, a contacting method using an optical fiber is introduced. The purpose of the method is to achieve a reference of the non-contacting method and to provide the educational material of fiber optics to students. Finally, other apllications of the laserbeam reflection method are explained.

\section{WAX CYLINDERS AND EDISON-TYPE PHONOGRAPH}

For the first step of the reproduction of sounds from old phonographic wax cylinders, properties of wax cylinders and an Edisontype phonograph should be understood. The dimensions of the most popular type of wax cylinders are $55 \mathrm{~mm}$ diameter and $105 \mathrm{~mm}$ long. About 400 turns of sound grooves with $254 \mu \mathrm{m}$ pitch are cut on their surface. Normally, the rotational velocity at reproduction was 140 $160 \mathrm{rpm}$. Therefore, about 2-3 minutes of recording were possible. By using the Edisontype phonograph with a cutting stylus, the

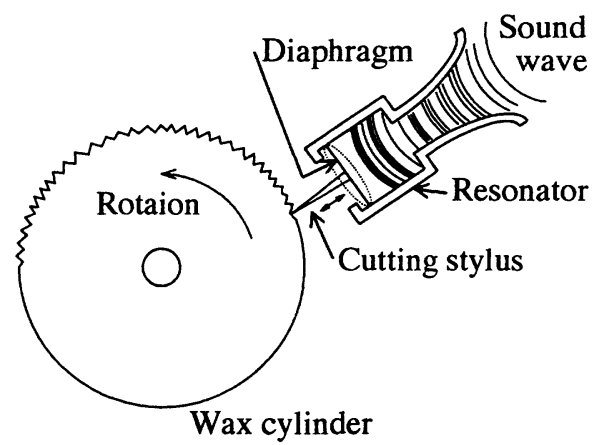

Figure 1. Schematic diagram of the procedure to record sounds on the wax cylinder.

recording was made on wax cylinders by a direct cutting method. Since the cutting energy was supplied only by the sound pressure transmitted to the stylus through the diaphragm, the depth of sound grooves were $\approx 50 \mu \mathrm{m}$ at most. This situation is shown in fig. 1. Because of the principle of the direct cutting method, the depth of sound grooves is directly related to the sound signal itself. In other words, a radial variation in the bottom of sound grooves represents sound signals. Therefore, the reproduction of sounds from old wax cylinders is conducted from a sensitive detection of microscopically fine structures on a macroscopically curved surface.

By using an Edison-type phonograph with a reproduction stylus, the reproduction of sounds from wax cylinders can be performed. There are some Edison-type phonographs which are still available in museums and in personal posessions. Although it is an easy way to use those phonographs for the reproduction, their stylus pressures are normally over 50 grams and the reproduction by those phonographs results in destructive damages on sound grooves. In fact, wax cylinders, grooves of which were worn out by too many counts of reproductions, are found now but no information can be obtained from them. The non-destructive method for the reproduction of sounds from old wax cylinders are, hence, important to be developed. 


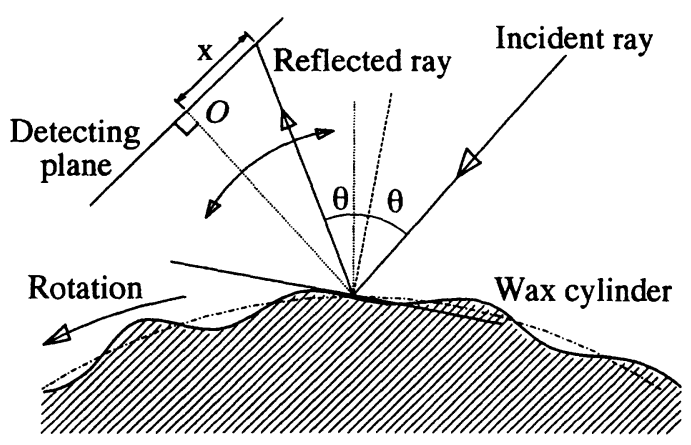

Figure 2. Intersection of the wax cylinder and the schematic 2-D diagram of the laserbeam reflection method.

\section{LASER-BEAM REFLECTION METHOD}

The principle of the laser-beam reflection method and its benefits for teaching to students are explained in the former part of this section. In the latter part, problems and shortcomings of this method, which are mainly due to the wave property of light, are described.

\subsection{Principle of the laser-beam reflection method}

Figure 2 shows an intersection of a wax cylinder and the principle of the laser-beam reflection method. An optical ray is incident on a groove cut on the surface of the wax cylinder and is reflected according to the law of reflection. The reflected ray reaches the detecting plane placed perpendicularly to the optical axis of reflection, with the origin centered at the point of intersection with the optical axis. With the rotational movement of the wax cylinder, the angular direction of the reflected ray varies according to a variation in the radial depth of sound grooves. This method seems to be based on the optical magnification of the displacement. By placing a position sensitive device (PSD) in the detecting plane, the recorded sound signal is detected as an electronic signal of the displacement of the reflected ray. While a 1-dimensional PSD is sufficient for this method in principle, a 2dimensinal PSD, S1300 (HAMAMATSU Photonics), is used because of the tracking error problem to be mentioned later. Figure 3

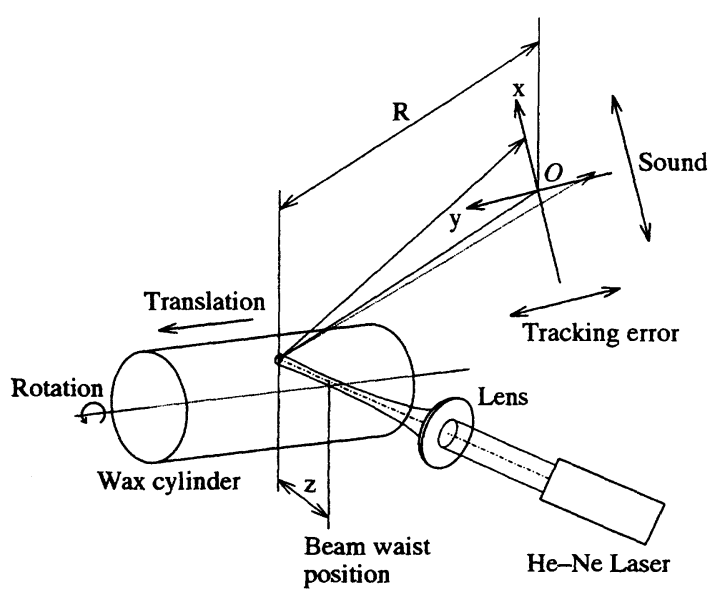

Figure 3. Schematic diagram of the procedure to reproduce sounds from the wax cylinder.

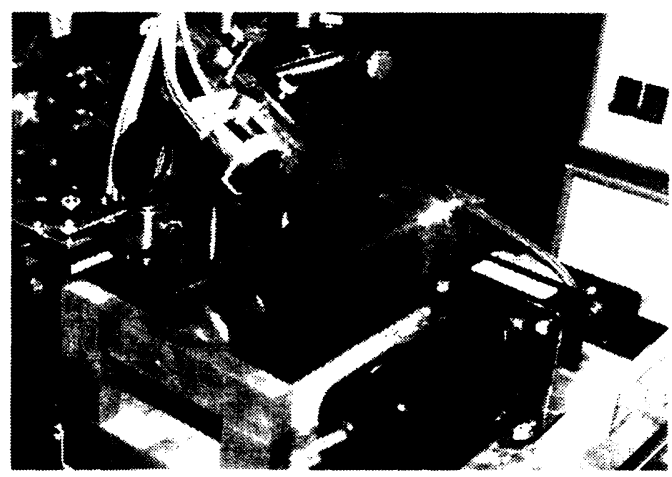

Figure 4. Photograph of the whole system except for the laser source.

shows the schematic 3-D diagram of the laserbeam reflection method to reproduce sounds from wax cylinders. In the figure, the rotation and translation movements are established by a DC geared motor and a pulse-motor stage, respectively. The rotation system is mounted on a pulse-motor stage and the translation movement is adjusted to produce the same increment with the pitch of the sound grooves by detecting the rotation speed using a rotary encoder mounted on the rotation axis. The 


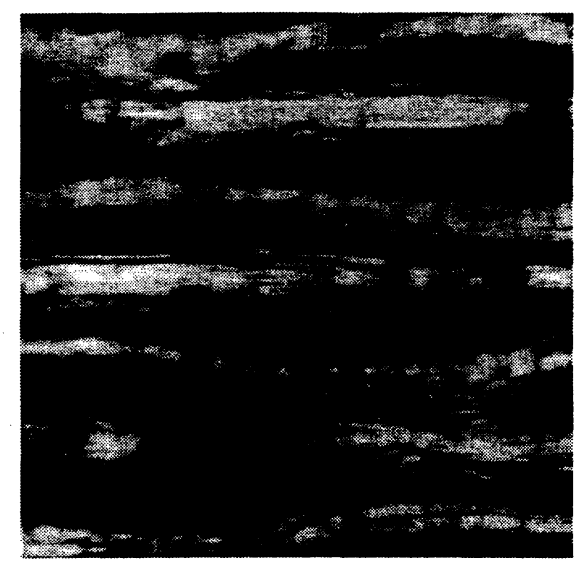

Figure 5. Microscopic photograph of sound grooves.

whole system without the laser source is shown in fig. 4.

The method is easily acceptable by students because it is based only on the geometrical optics. Furthermore, the optical configuration required is so simple that the setting up of the optical system is a good training material in optics education. Misalignments or incorrect arrangements bring no sounds. When the optical elements are arranged properly, sounds reproduced make students exciting since the sounds they can hear were recorded about one century ago. Students can gain a lot of the basic skill from this experience.

\subsection{Extension to the diffraction theory}

Although the principle of the laser-beam reflection method is explained by using the geometrical optics, there are some problems and shortcomings which will be mainly explained by the diffraction theory.

The bad preservation conditions of wax cylinders caused not only physical but also chemical and biological damages to sound grooves and resulted in producing roughened surface profiles on them. Even in the case of wax cylinders preserved in good conditions, their surface profiles are rough compared with the wavelength of light used for the reproduction. A microscopic photograph of a surface of a wax cylinder is shown in fig. 5 . The reflected light waves from the wax cylinder, therefore,

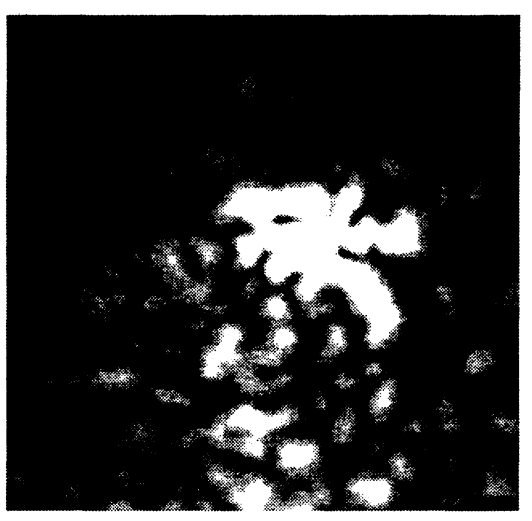

(a)

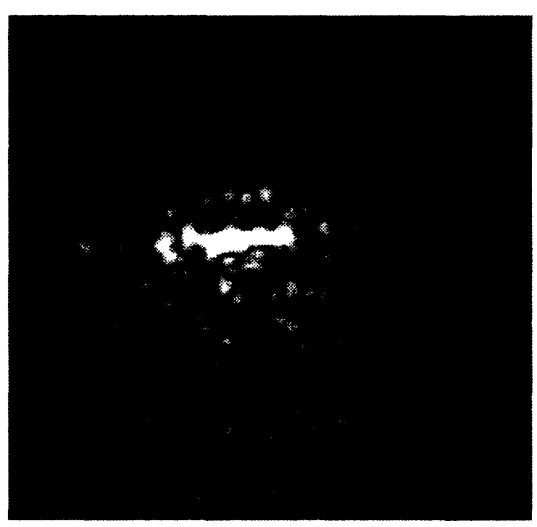

(b)

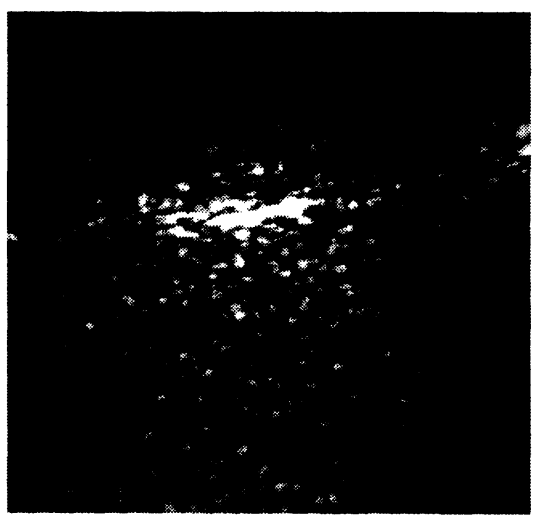

(c)

Figure 6. Photographs of reflected rays at the detecting plane with illuminating laser beams of three different radii of (a) $30 \mu \mathrm{m}$, (b) $60 \mu \mathrm{m}$, and (c) $110 \mu \mathrm{m}$. 


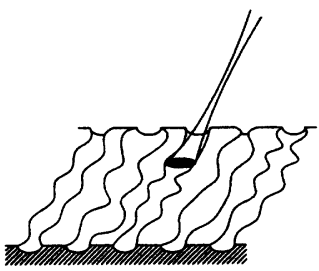

(a)

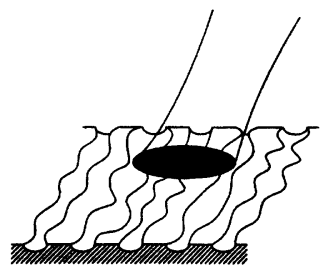

(b)

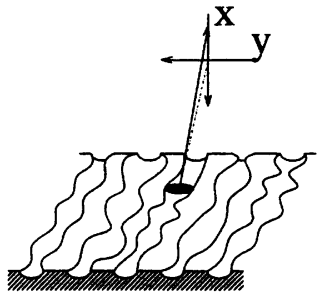

(a)

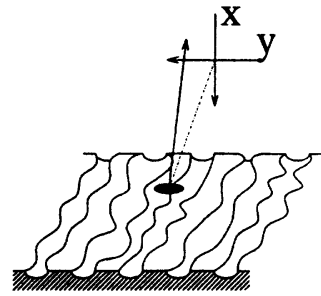

(b)
Figure 7. (a) suitable illumination and (b) overillumination on the grooves of the wax cylinder.

interfere each other and a speckle pattern can be observed in the detecting plane. Since a PSD's output is proportional to a mean moment of the light power distribution being incident on the sensitive area of the PSD and the speckle pattern varies dynamically with a change in the illuminating area of sound grooves, a variation of the speckle pattern brings the unpreventable noises during the reproduction. The speckle patterns observed in the detecting plane are shown in figs.6(a)-(c) with three different out-of-focus distances $z$. This phenomenon helps students to realize the wave property of light. The relation between the distance $z$, which gives rise to a variation in the illuminating spot size, and the average speckle size in the detecting plane is directly observable. In addition, a variation in the illuminating spot size has a great effect on the quality of sounds reproduced. The fact that the adjustment of an optical arrangement is instantly reflected on the quality of reproduced sounds provides students with fascinating experience.

The size of the illuminating beam spot has another important role in the reproduction of sounds. Figures $7(a)$ and (b) show two cases of the schematic relation between the spot size and the sound grooves. In fig. 7(a), the spot size is adequately adjusted and it traces only one portion of the groove during the reproduction. On the contrary, in fig. 7(b), the spot size is so large that the spot covers two or more adjacent parts of the grooves and simultaneously traces them. This causes an echo effect during the reproduction and degrade the quality of reproduced sounds.
Figure 8. Optical rays reflected from the grooves (a) without and (b) with the tracking error.

\section{IMPROVEMENTS OF THE LASER-BEAM REFLECTION METHOD}

\subsection{Compensation of tracking errors}

Position of the beam spot with respect to the sound grooves are shown in figs.8(a) and (b). In the ideal case of fig. 8(a), the tracking is correctly performed and a deviation of the reflected beam along the $y$-direction does not occur and, hence, a 1-D PSD is sufficient for the detector. On the other hand, in fig. 8(b), the beam is incident on the sound groove with the tracking error and the reflected beam is shifted along the $y$-direction. To avoid the effect of tracking errors, a 2-D PSD is used. Although the reflected beam varies along the $y$-direction on the PSD's surface, only a variation of signals along the $x$-direction is used to reproduce sounds. In this manner, the reproduction is performed as long as the reflected beam varies inside the sensitive area of the PSD. Furthermore, to compensate the tracking errors, a 2D PSD is again used. The output signal along the $y$-direction from the 2-D PSD can be used for the compensation signal of the tracking error by adjusting the translation speed of the pulse-stage on which the rotating wax cylinder is mounted. Regardless of the existence of tracking errors, only signals along the $x$ direction is available for the reproduction of sounds.

\subsection{Reduction of stray light}

In principle, the PSD detects the mean position of the intensity distribution over its detecting surface. Since the output from the 


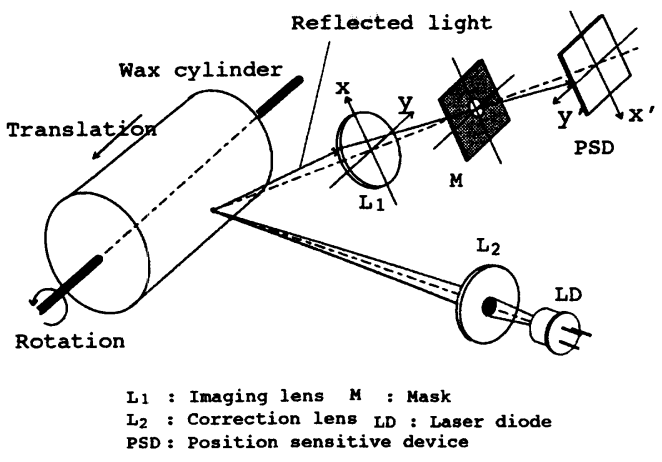

Figure 9. Schematic diagram of the system to reduce the effect of the stray light.

PSD is fed into an electronic circuit which is designed to compensate multiplicative variations of the intensity distribution, reproduced sounds are insensitive to variations in the laser power and the reflectance of the cylinder surface. On the other hand, additive variations of the light incident on the PSD, such as the stray light, do affect the reproduced sounds and the reduction of such an effect is desirable.

A simple way to this end is to insert an additional optics between the wax cylinder and the PSD as shown in fig. 9. This optics consists of a lens and a diaphragm with a pinhole placed in the image plane of the illuminating spot. Since the image of the illuminating spot acts as the same way with the illuminated portion of the groove, the PSD detects almost the same signal as in the case of fig. 3. Hence, by covering the part from the lens to the PSD, a considerable rejection of the stray light can be achieved.

In fig. 9, another improvement primarily intended to the compactness of the system is shown. Use of a laser diode made a great progress in the compactness of the system. This is also a good educational material for students to understand differences between a gas laser and a laser diode.

\section{OTHER IMPROVEMENTS}

\subsection{Incoherent light source}

To avoid the noise caused by speckles, it is natural to consider the use of an incoherent light source for illumination. Although here are

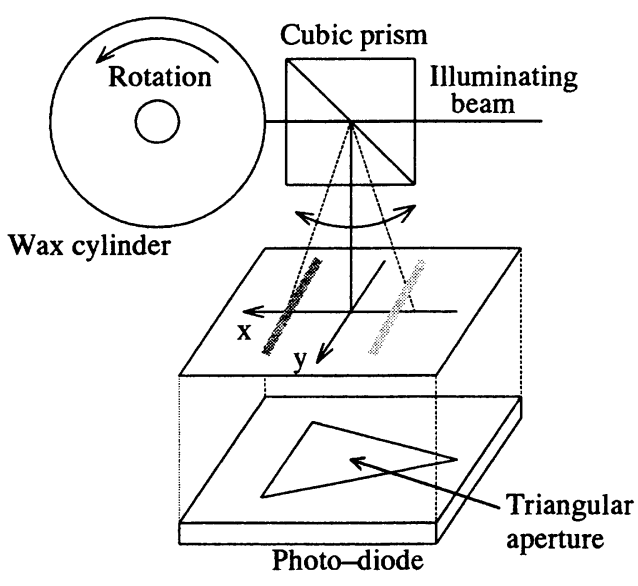

Figure 10. Schematic diagram of the optical configuration to use a photo-diode.

many kinds of incoherent light sources which are available now, it would be a rather difficult work to create a small spot for our purpose with a simple optical configuration. A difference between the coherent and incoherent lights is one of the important subjects in the diffraction theory. Students trying to set up the optical configuration to produce a small spot size with an ordinally incoherent light source can realize that any speckle patterns will not be observed in the detecting plane and that a small spot size of the light is hardly achieved as was easily done by using a laser.

A way of achieving a small spot with simple optical elements is to use an infrared pointsource LED such as LSB879B (OPTRANS Corp.), with the central wavelength of $880 \mathrm{~nm}$. Since this LED has a circular radiation area of $150 \mu \mathrm{m}$ diameter and also has uniform radiation over the entire circular area, the beam spot imaged on a sound groove using an objective lens of the microscope is small and fine enough to trace a single sound groove in experiments. The PSD used has a sufficient sensitivity in the infrared wavelength of the LED. Hence, except for this light source, the same optical configuration with that used in the laser-beam reflection method could be employed. No speckle patterns can be observed in the reflected beam spot and the quality of the resulting reproduced sounds are increased 


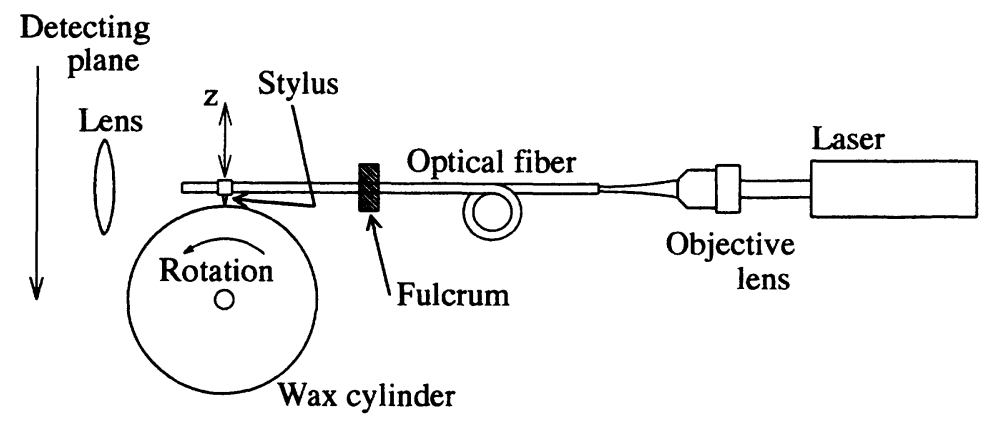

Figure 11. Schematic diagram of the contacting method using an optical fiber with a stylus.

from the viewpoint of the $\mathrm{S} / \mathrm{N}$ ratio. In spite of this benefit, however, the light power is at most $3 \mathrm{~mW}$ and is less compared with the laser used in the original laser-beam reflection method and the invisibility of the light produces a hard problem in both a demonstration to students and a setting up of optical elements by themselves.

\subsection{Use of a simple photo-diode}

An ordinaty photo-diode was tried for the purpose of a replacement with a PSD. Although the PSD is a powerful detector for our experiments, it is so expensive that the use of the PSD raises up the system cost.

To accomplish a linearly proportional output of the photo-diode with the beam position reflected at a sound groove, two idea were employed. One is a perpendicular illumination. When such an illumination is performed on sound grooves, a reflected beam reveals a shape of a sheet beam elongated along the $y$ direction and varies along the $x$-direction accroding to a variation of the sound groove. The other is to use a photo-diode with a triangular aperture. This situation is shown in fig. 10.

The new detector operates successfully unless the tracking errors exist. Because this detector is insensitive along the $y$-direction, the other detector is required to detect the tracking errors. This makes the optical configuration more complicated. A trade-off of the cost and the complexity of optics are troublesome in education.

\subsection{Contacting method using the fiber optics}

So far a contacting method has not been taken into consideration because of damages on sound grooves it may cause. However, it is still true that the contacting method is the most proper way to reproduce sounds from wax cylinders because it can most faithfully trace sound grooves on the reproduction. In the case of an Edison-type phonograph, vibrations of the stylus were transmitted to the diaphragm and a magnification of reproduced sounds were performed only by an acoustic horn. The low stylus pressure results, therefore, in reproducing low sounds, while destructive damages occur when the stylus is used with the heavy pressure. Nowadays, however, an electronic magnification is available and, hence, the contacting method with the low stylus pressure may also be useful. It is also necessary for this study to construct a reproduction system using the contacting method with negligible damages because it may provide a reference to the non-contacting method.

Various styluses were examined for the contacting method. Although a set of audio pickups was considered as one of the most suitable ones for this purpose, the stylus of an audio pickup was so sharp to be employed for wax cylinders that it detects very fine surface structures of the sound groove and gives rise to noisy sounds especially in a higher frequency domain. Furthermore, since currently available audio pickups are manufactured for stereo recordings on disk records, their me- 


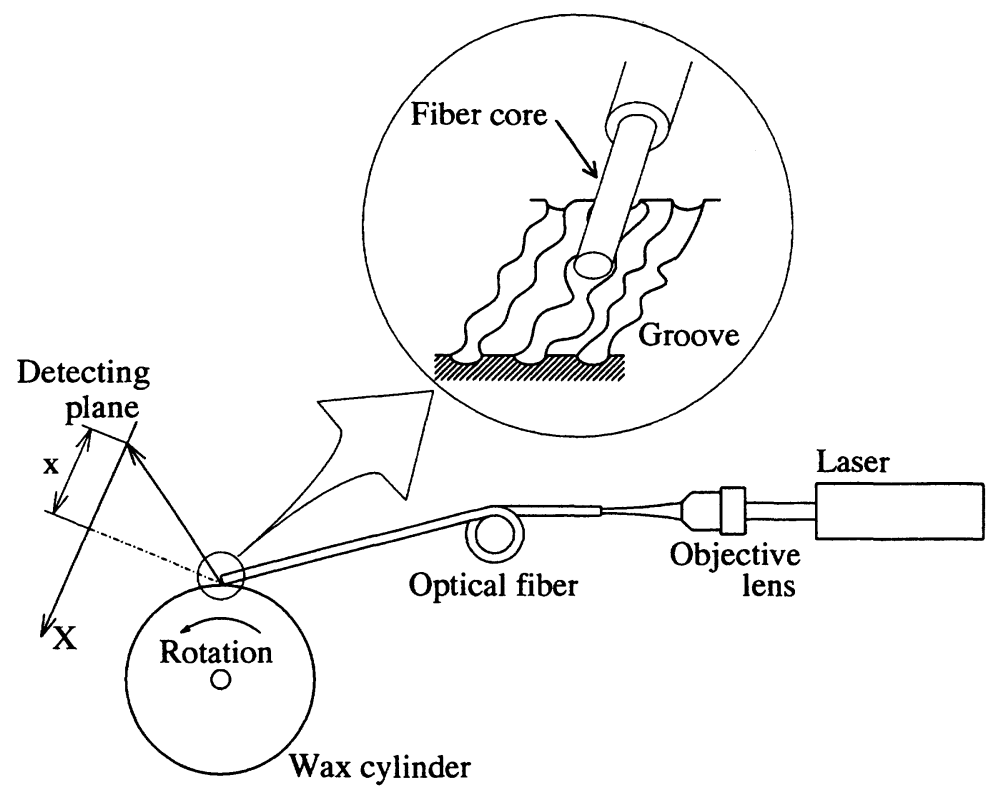

Figure 12. Schematic diagram of the contacting method using an optical fiber without a stylus.

chanical and electronical specifications are not adequate for the reproduction of sounds from old wax cylinders.

Next a method using an optical fiber with a stylus is examined. This method is introduced for two purposes: one is to achieve a contacting method with the low stylus pressure, and the other is to teach optical fibers to students. Students can get a skill of handling optical fibers: how to cut, how to hold, knowledge of entrance and exit pupils, and others. Figure 11 shows a schematic diagram of this method. The stylus moves in the $z$-direction with the rotation of the wax cylinder and the light emerging from the optical fiber is incident on a PSD. The output of the PSD is directly proportional to the recorded sounds. The contacting method with the low stylus pressure $(\approx$ a few grams) is principally achieved by using an optical fiber. However, the quality of reproduced sounds still depends mainly on the stylus itself and unforeseen noises arise from mechanical vibrations of the optical fibers laid on the optical bench. The fiber picks up various vibrations, for example, from the pulsemotor stage and from the DC geared motor, and these vibrations appear as noises in the reproduction of sounds. Vibrations due to the stylus movements also cause a mechanical resonance of the optical fiber. Therefore, further investigations on mechanical vibrations are required for this method to be completed.

A method using an optical fiber without a stylus is finally examined. The core of a multimode optical fiber with the $150 \mu \mathrm{m}$ diameter was found to be a good stylus for the reproduction of sounds from old wax cylinders. Figure 12 shows a schematic diagram of this method. The tip of the fiber core is placed in a sound groove. The laser light guided into the fiber core emerges from the fiber tip and illuminates the sound groove. The reflected light propagates toward the detecting plane and is incident on the PSD. This method may be considered as a modification of the laser-beam reflection method. By using the optical fiber, a problem of the groove tracking is resolved and a stable illumination is achieved. However, a problem of the speckle noise caused by using the laser light has remained. Therefore, the sounds reproduced by using this method quite resembles those reproduced by the laser-beam reflection method. 


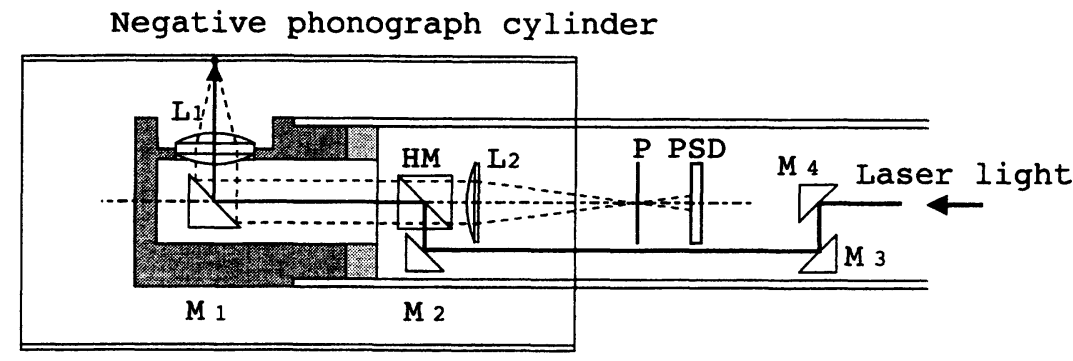

Figure 13. Compact optical head inserted into the negative cylinder. The illumination optics consists of mirrors $M_{1}-M_{4}$, a half mirror $H M$ and a lens $L_{1}$, while the detection optics consists of $\mathrm{L}_{1}, \mathrm{HM}$, a lens $\mathrm{L}_{2}$, a diaphragm with a pinhole $\mathrm{P}$, and a 2-D PSD.

\section{APPLICATION TO OTHER TYPES OF RECORDS}

\subsection{Disk records}

Having learned the method for the optical reproduction of sounds from wax cylinders, students may wonder if this technique is applicable also to old disk records. They will soon realize that the grooves of disk records are different from those of wax cylinders. Namely, the grooves of disk records are winding in the disk plane and the sound information is encoded in this lateral displacement of the groove. In contrast with that, the sound information is encoded in the vertical variation of the groove for wax cylinders. This means that the laserbeam reflection method cannot be applied directly to disk records.

However, a careful observation of the reflected beam from the groove of a disk record will reveal that a horizontal variation of the groove certainly modulates the reflected beam. As long as the size of the illuminating spot is not so large as the groove width, the illuminated part of the groove is considered to be straight. Such a straight structure produces again a straight structure in the reflected optical field according to the diffraction by the groove. This linear diffraction pattern is perpendicular to the direction of the illuminated part of the groove. Hence, in principle, the local direction of the sound groove can be detected by sensing the direction of the diffraction pattern. This leads us to the laser diffraction method for the reproduction of sounds from disk records. Details of this method are described in a previous article. ${ }^{2}$ Here it is only noted that this crude method is applicable only to monaural disk records and that the tracking of the laser beam along the groove is much more difficult than the laser-beam reflection method.

\subsection{Negative cylinders}

Another interesting extension is briefly introduced here though it is not straightforward for students to actually confirm it. Recently, the authors have come to know that there are many negatives of wax cylinders in Germany. The negative was made by plating a wax cylinder with copper and, then, by melting down the original wax cylinder. Thus, the sound information is transferred to the inside wall of the metallic cylinder as vertical variations of a narrow bank of metal. Since the original wax cylinders were lost in this process and the method of reproducing a replica cylinder from the negatives has been lost, those negative cylinders have been left for a long time without reproduction of sounds from them. It is noted that any contacting method using a stylus cannot reproduce sounds from such records, because sound signals are not included in a concave structure but in a convex one.

It is found that the laser-beam reflection method is also effective to those negative cylinders. To apply it to the negatives, however, some modifications were required to the instrument. First, an optical system must be small enough to be inserted into the negative 
(fig. 13). The holding and driving mechanisms were also altered so that the negative is held from the outside by a larger cylinder which is rotated and translated axially during the reproduction. With these modifications, the laser-beam reflection method played negative cylinders successfully. It is interesting to note that, in general, the sounds from the negatives are much better than those from old wax cylinders because the negatives are considered to have been produced soon after their recordings while the wax cylinders left now have been played very many times by stylus players. Details about the application of the laser-beam reflection method to the negative cylinders will be described in a forth coming paper.

\section{CONCLUSION}

The basic method and some variants of it for the reproduction of sounds from old wax cylinders were demonstrated in relation to the educational viewpoint. The facts that a change of optical configurations and/or parameters is directly reflected in reproduced sounds and that students can check them by their hearing provide a great motivation for students.

It was shown first that the laser-beam reflection method is an attractive material for teaching basic elements of optics. In a primary step, students can understand its principle from the geometrical optics. Then, they will soon recognize that it is necessary to use the diffraction theory to explain problems encountered in the process of experiments. The optical setup is not so complicated nor so sensitive and, hence, students can easily adjust optical elements to see their effects on the reproduced sounds in real time.

Next, we have demonstrated that the laserbeam reflection method affords a room for improvements, which provide opportunities for students to analyze optical problems, to modify the original optical system, and to devise alternate optical systems. Each method involves different optical subjects in elementary optics, and discussions and attempts on them will help students to cultivate their ability in optics.

Finally, the importance of this subject should be mentioned. The Edison's invention of phonographs was the first of the audiorecording in the world. It had spread rapidly and widely all over the world and many kinds of sounds were recorded in lots of wax cylinders. After about a half century from the invention, the Edison's phonograph was gradually declined and finally replaced by disk records. There remain now numerous old wax cylinders, many of which are valuable from the viewpoint of linguistics, folklore and music history. Non-destructive reproduction of sounds from those old wax cylinders gives the technological assistance to researchers in those fields. This cultural importance would be another effective motivation to encourage students to learn this method and to try further improvements of it.

\section{REFERENCES}

1. T. Iwai, T. Asakura, T. Ifukube, and T. Kawashima, "Reproduction of sound from old wax phonograph cylinders using the laser-beam reflection method," Appl. Opt., 25, (5), 597-604 (1986).

2. J. Uozumi and T. Asakura, "Reproduction of sound from old disks by the laser diffraction method," Appl. Opt., 27, (13), 26712676 (1988). 